\title{
Valores sociales y ejercicio físico en escenarios universitarios ${ }^{*}$
}

\author{
Edwin Arcesio Gómez Serna**
}

Recibido: 14 de septiembre de 2012

Revisado: 9 de octubre de 2012

Aceptado: 5 de diciembre de 2012

\section{Resumen}

Diversos antecedentes indican la incidencia de las actividades deportivas y físicas en el desarrollo y la consolidación de hábitos asociados con algunos valores sociales. En este orden de ideas, el siguiente artículo presenta los resultados de revisión teórica y conceptual que aportaron al marco teórico del proyecto Deporte y Valores: "Influencia del ejercicio físico en el afianzamiento de valores sociales". En este proceso de investigación se interpretó la relación entre la práctica del ejercicio físico en los gimnasios universitarios y algunos valores de carácter social considerados como positivos. A partir de una propuesta de tipo hermenéutico y siguiendo metodologías, instrumentos y procedimientos de análisis e interpretación de carácter cualitativo, se identificaron algunas tendencias teóricas y conceptuales alrededor de esta temática.

Palabras clave: ejercicio físico, valores, gimnasio, universidad.

* Este artículo presenta una reflexión de carácter teórico y conceptual sobre la temática en cuestión y hace parte del proceso de investigación "Influencia del ejercicio físico en el afianzamiento de valores sociales", realizado durante 2012 en la Facultad de Cultura Física, Deporte y Recreación, y avalado por el Comité de Investigación de la Facultad. Participaron en calidad de auxiliares de investigación y como opción de trabajo de grado los estudiantes Julián Enrique Aponte Mogollón, José Miguel Méndez Acuña, Tatiana Garzón Sánchez y Jessica Alejandra Valencia Aranguren.

** Licenciado en Filosofía y Letras. Magíster en Educación y Desarrollo Humano. Docente de la Facultad de Cultura Física, Deporte y Recreación de la Universidad Santo Tomás. Correo electrónico: edwingomez@usantotomas.edu.co 


\title{
Social values and physical exercise in university environments
}

\begin{abstract}
Various records indicate the impact of sports and physical activities in the development and consolidation of habits associated with some social values. Along this line, the following article presents the results of theoretical and conceptual review provided to the theoretical framework of the Sports and Values project: "Impact of physical exercise in the strengthening of social values". This research process interpreted the relationship between practicing a physical exercise in university gyms and some social values considered as positive. From a hermeneutic type proposal and following methodologies, tools and qualitative analysis and interpretation procedures, some theoretical and conceptual trends on this topic were identified
\end{abstract}

Keywords: physical exercise, values, gym, university.

\section{Introducción}

Diversos estudios en Latinoamérica presentan la relación entre los procesos de formación en valores y la práctica de la actividad física, el deporte y la recreación. La revisión teórica realizada dentro del proyecto indicó que, desde la perspectiva del deporte y la actividad física, esta temática se ha investigado desde tres grandes campos: la psicología del deporte, la sociología del deporte y la educación física.

La psicología del deporte se ha concentrado en demostrar, desde perspectivas de corte positivista y bajo metodologías empíricas, la directa relación entre ciertas prácticas deportivas y recreativas y el desarrollo de actitudes y comportamientos asociados a valores como el respeto, la cooperación, la autoestima, etc. De igual forma, se encuentran gran cantidad de estudios que desarrollan la relación entre ciertos comportamientos asociados a 
valores: compromiso, disciplina, trabajo en equipo, etc., y su incidencia en el rendimiento deportivo, el logro de objetivos y el éxito deportivo.

Con respecto a la sociología del deporte, se identificó un énfasis en la producción investigativa sobre el desarrollo y estudio de los procesos de socialización primaria y secundaria que se dan en diversos escenarios en donde el deporte y la actividad física se practican y la forma en que aportan a otros procesos asociados, como la comunicación, la construcción de vínculos afectivos, etc. De igual forma, se busca comprender la relación de diversos agentes socializadores en la configuración de las relaciones que dan pie a los procesos formativos. Por último, se identificó otro grupo de trabajos concentrados en explorar, desde una perspectiva sociológica, problemáticas relacionadas con los valores y asociadas a las prácticas deportivas, como el juego limpio, la trampa o el dopaje.

En el campo de la educación física, el énfasis se concentra en estudios y reflexiones que establecen una clara intención frente a la función educativa del deporte y la recreación en los procesos formativos del contexto educativo. En la misma línea de los campos explorados, es claro que se asumen las diferentes prácticas deportivas y recreativas como potenciadoras de comportamientos asociados a valores de diverso tipo. En este orden de ideas, todo proceso de aprendizaje se fortalece $\mathrm{y}$ crece mediante experiencias prácticas, entre ellas el ejercicio físico: "Numerosos autores han señalado que la actividad física y el deporte pueden resultar excelentes medios para transmitir valores personales y sociales, tales como respeto, autocontrol, autoestima, empatía, esfuerzo, autonomía, cooperación, ayuda a los demás, hábitos saludables o liderazgo" (Pardo, 2008, p. 7).

La anterior revisión muestra un claro hilo conductor: el impacto del ejercicio, el deporte y la actividad física para el afianzamiento de valores depende de tres elementos: el entorno en donde se desarrolla dicha actividad, los actores y el tipo de prácticas realizadas por los sujetos. De igual forma, es claro que la práctica deportiva aporta al ser humano importantes beneficios físicos, psicológicos y sociales: los estudios muestran la importancia de la formación en valores en el campo deportivo y de la actividad física; sin embargo, aún no es claro cómo dicho proceso se desarrolla en un contexto que comporta características educativas, debido a su ubicación en el contexto universitario, como elementos propios del ejercicio físico, debido a su regularidad y nivel de exigencia. 
Esta revisión evidenció la pertinencia de realizar la anterior investigación y buscó resolver la siguiente pregunta: ¿cómo influye la práctica de ejercicio físico en el desarrollo y afianzamiento de valores sociales tales como el orden, el trabajo en equipo, el compromiso y la disciplina, en un escenario de carácter educativo y deportivo como los gimnasios ubicados en instituciones universitarias?

\section{Método}

Metodológicamente, la investigación se desarrolló con el enfoque cualitativo y desde un paradigma de tipo hermenéutico, en donde los procesos de comprensión e interpretación de los fenómenos se realizaron en un contexto histórico y cultural específico. El propósito fundamental de los procesos investigativos de corte hermenéutico en las ciencias sociales es comprender las experiencias colectivas humanas en contextos específicos. En palabras de Hoyos y Vargas (1996), es en el mundo de la vida y en la experiencia intersubjetiva de los sujetos en donde se realiza la interpretación. Dicho enfoque permite, desde el punto de vista metodológico, una compresión de lo social en términos de reconstrucción del sentido que tiene, para los sujetos, la vivencia de cierta situación en un contexto histórico y cultural específico, dimensión que se construye estableciendo un vínculo entre los contextos sociales y la lógica investigativa.

Por esto el proyecto estableció cuatro procedimientos, a saber: a) una revisión de tipo teórico y de antecedentes en relación con la temática; b) la construcción y aplicación de los instrumentos para la recolección de la información enfocados en dos técnicas básicas: la observación participante y la entrevista semiestructurada (Taylor y Bogdan, 1987); c) el análisis de la interpretación recolectada y sistematizada; d) la construcción de un texto de tipo comprensivo que dé cuenta del análisis y de la interpretación. Cada uno de estos procedimientos se alimentaba en forma constante tanto de la revisión y el análisis permanentes de los textos, como de la vivencia y experiencia de los coinvestigadores.

Con estos criterios, la revisión que dio origen al marco teórico del proyecto siguió la propuesta de Souza (2008) para construir un estado del arte de carácter interpretativo alrededor de las temáticas deporte y 
valores; dicho ejercicio buscó conocer y sistematizar la producción científica (artículos científicos) alrededor de ellas. Esta exploración bibliográfica permitió elaborar una reflexión sobre los procesos investigativos y de reflexión en el contexto latinoamericano alrededor de las temáticas y la revisión de antecedentes identificó una clara falencia en los procesos investigativos desde una perspectiva cualitativa, no solo dentro del grupo y la línea de investigación, sino en el contexto regional, ya que se encontró que gran cantidad de investigaciones se fundamentan desde el paradigma positivista.

La orientación de esta investigación, desde una perspectiva cualitativa y fundamentada en un ejercicio de tipo hermenéutico, aporta en el desarrollo y la consolidación de estrategias, técnicas e instrumentos que aporten al acervo investigativo de dichas temáticas. De igual forma, existe un interés específico por evidenciar la importancia y el aporte que el aprovechamiento de espacios de interacción, diferentes al aula de clase, tiene en los procesos de formación en valores. Estos procesos deben trascender de la instrucción de conceptos de carácter teórico hacia la apropiación conceptual y praxiológica de los valores sociales, para lo que es necesario vincular la teoría con la práctica. Es aquí donde la participación en una actividad presenta un claro etos, tanto de carácter individual como grupal.

Este ejercicio permitió identificar qué se ha investigado hasta ahora y los aportes reflexivos de diversos estudiosos del tema, teniendo en cuenta su contexto y los criterios y puntos de vista sobre los que gira dicha producción. La autora en cuestión, Souza (2008) aclara que para realizar este tipo de ejercicios es fundamental delimitar claramente tanto los campos temáticos como algunos criterios de inclusión-exclusión de los documentos por revisar. Para lograr esto, se hizo una búsqueda en diferentes bases de datos, revistas y textos de carácter académico en donde se evidenciaran las temáticas valores y deporte, estableciendo una clara relación entre estos dos conceptos. Frente a criterios de exclusión, solo se estimó que la producción proviniera de una fuente de carácter académico -no necesariamente científico-, ya que también se tuvo a acceso a documentos relacionados con la política pública y proyectos educativos de desarrollo. 


\section{Resultados}

Con respecto al ejercicio físico, concepto nuclear en esta propuesta investigativa, Sánchez-Bañuelos (1996), citado por Gutiérrez (2004), lo define como todo acto físico motriz intencionado y programado, específico y sistemático, con un objetivo definido. Por lo tanto, el ejercicio físico que carezca de rigurosidad, objetivos y un diseño en su programación, planificación y estructura, se considera una actividad física. Es por esto que un adecuado ejercicio físico, realizado con frecuencia, intensidad y duración adecuadas, fomenta comportamientos favorables para la salud y -en opinión de los investigadores-fomenta valores como la disciplina y el orden.

Hoy, los gimnasios se convirtieron en una gran alternativa para realizar ejercicio físico y en una salida positiva a la minimización de la motricidad que arrojan los avances tecnológicos, las dinámicas urbanas, y los problemas asociados a los malos hábitos alimenticios y al sedentarismo. Y aunque el ejercicio físico que se realiza en los gimnasios posea gran popularidad al estar orientado en un alto porcentaje a la estética, convirtiéndose en una panacea cosmética, posee factores determinantes para el mejoramiento de los niveles de salud, que afecta directamente el bienestar físico, psíquico y social de quienes lo practican regularmente.

Cabe aclarar que no se identifica, en la revisión realizada, al ejercicio físico en los gimnasios como orientado por objetivos educativos. La mención más cercana a este punto se refiere al desarrollo de hábitos de vida saludable, ya que se percibe una sensación general de bienestar, lo que valora de manera positiva al ejercicio físico. A nivel psicológico, es importante señalar que el ejercicio proporciona distracción, diversión, cambios en las emociones y conductas que mejorarán el estado anímico de toda persona. Igualmente, hay un incremento en la autoestima y conducta de cada persona debido a la reducción de tensiones y ansiedades.

A nivel social, y siguiendo a Gutiérrez (2004), se presentan los siguientes beneficios de la práctica del ejercicio físico en el gimnasio:

1. Adquisición de pautas de conducta y relación firme, encaminadas a la cooperación, amistad, comunicación.

2. Autocontrol en las reacciones emocionales que plantea lo cotidiano. 
3. Superación, esfuerzo imprescindible para el desarrollo, madurez personal y funcionamiento grupal.

4. Participación, donde el individuo abandona su egocentrismo y en ocasiones se convierte en líder.

5. Sociabilidad, expansión positiva de la persona respecto a quienes lo rodean.

6. Educación en la libertad y responsabilidad como las dos caras de la misma moneda.

7. Creación de espacios de respeto y tolerancia frente a la opinión de los demás.

El anterior trabajo investigativo hace referencia a la relación entre ciertos valores sociales y la práctica del ejercicio físico en el gimnasio; en este sentido, dicho trabajo presenta una clara congruencia con la presente propuesta investigativa. Sin embargo, aún no son evidentes, en la revisión realizada, los elementos de carácter deportivo y axiológico que establezcan una relación entre la práctica del ejercicio físico y los valores sociales; de igual forma, no es claro el proceso que lleva a dicho afianzamiento.

A partir de la identificación de la relación entre el ejercicio físico, el gimnasio y ciertos comportamientos sociales, se procedió a revisar la producción temática desde los tres grandes campos presentados en la introducción.

En el campo deportivo y soportado por la psicología deportiva, la investigación y producción del conocimiento sobre valores y deporte se concentra en el entrenador, en los procesos de entrenamiento y en la instrucción deportiva, etc. Desde esta perspectiva, se desarrollan hábitos, durante el acompañamiento al proceso de entrenamiento y preparación deportiva, que desembocan en el afianzamiento de ciertos valores sociales. Sin embargo, en lo revisado hasta ahora no se identifica claramente el aporte específico de un escenario asociado al ejercicio físico (el gimnasio) a esta temática ni mucho menos en la perspectiva de los procesos formativos propios de la universidad moderna. En esta línea de pensamiento, Torregrosa y Lee (2009, p. 71) argumentan que, al estudiar los valores, deberán considerarse conjuntamente otras condiciones, como el escenario de práctica, los pares, el proceso propiamente deportivo, etc. Los mismos autores concluyen que la práctica deportiva o física no influye -de forma directa- en el individuo y por lo tanto se requiere de un componente actitudinal, cognitivo y afectivo, 
es decir, este aprendizaje no es absolutamente autónomo. Gómez expone: "Una enseñanza deportiva educativa debe trascender el aprendizaje de lo meramente motriz para auspiciar otros ámbitos que tradicionalmente han sido soslayados como el cognitivo y, sobre todo, el moral" (2005, p. 91).

En esta misma línea de pensamiento, Boixadós, Valiente, Mimbrero, Torregrosa y Cruz (1998) presentan investigaciones en donde vinculan la formación de valores con la intervención de agentes socializadores, lo que incide en la calidad de la experiencia deportiva y la orientación del comportamiento de los jóvenes deportistas en su práctica deportiva, asociándola con el fairplay (juego limpio). Lo anterior muestra un nuevo enfoque para ver los valores, convirtiéndolos en un elemento de tipo informativo-evaluativo, que busca analizar la calidad de los procesos educativos en el ámbito deportivo. Sin embargo, ese trabajo se concentra en el estudio de jóvenes que participan en procesos de entrenamiento y no se refiere a otros escenarios, como el universitario, ni a una actividad específica, como la asistencia y permanencia en el gimnasio.

La Universidad Católica San Antonio de Murcia (España) adelantó en 2009 una serie de estudios a manera de recopilación, en la que se analizó la evolución de la aplicación de la psicología en el deporte y su impacto en el desarrollo de valores, mostrando resultados significativos con el tiempo. Olmedilla, Ortega, Garcés, Jara y Ortín (2009) presentan los resultados de su revisión; sin embargo, reconocen la falta de desarrollo en estas temáticas desde una perspectiva eminentemente cualitativa, debido -según los autores- a la "competición investigadora" que valora en alto grado los aportes de tipo cuantitativo y que utiliza metodologías de claro corte positivista.

La revisión muestra que, aunque se investiga y produce en esta temática, no se identifican aportes investigativos específicos asociados con el desarrollo de valores en el contexto universitario, ya que, desde esta perspectiva, los antecedentes revisados se circunscriben al trabajo, a los escenarios y a los actores enmarcados en los procesos de entrenamiento.

Otra línea de pensamiento e investigación sobre la relación deporte y valores se enfoca desde la perspectiva propiamente educativa, los escenarios y procesos relativos a la educación física. Los autores revisados expresan que en la actualidad existe una pérdida (o transformación negativa) considerable de valores, lo cual resulta ser uno de los principales desencadenantes de conductas negativas en niños y jóvenes. Flores y Zamora (2009) 
atribuyen este fenómeno, en parte, a deficiencias en la enseñanza de los valores sociales primordiales que deberían promoverse en la familia y en la escuela, razón por la que se aproxima al tema del potencial que tiene la educación física como medio para apoyar y promover el desarrollo de valores sociales positivos y cómo estos contribuyen a la construcción del carácter, la personalidad y la forma de pensar.

Este planteamiento se sustenta en las características especiales que tiene la educación física frente a otras áreas del conocimiento, en específico el claro énfasis praxiológico. Los autores delimitan su esfera conceptual y sus procesos investigativos al análisis con el fin de medir o evidenciar claramente la función que tiene la educación (física), desde sus diferentes escenarios, en el desarrollo y afianzamiento de valores sociales, lo que redunda en un aporte social de impacto significativo. Ahora bien, esto indica que el ejercicio de enseñanza-aprendizaje dentro de una clase de educación física lleva implícito un componente valorativo que se transmite de forma grupal y que, aunque se aprende de forma individual, tiene una aplicación social, producto de la interacción de los niños en el escenario educativo.

En este orden de ideas, autores como Ruiz y Cabrera establecen una directa relación entre estos dos escenarios, el práctico y el conceptual:

Las prácticas (educativas en la clase de educación física) que se organicen deben buscar el desarrollo integral, el desarrollo del cuerpo y de la mente, de la autoestima, el sentido ético y moral, de responsabilidad, de autonomía, de superación y de relación y aceptación de los demás, aceptación de las normas, de cooperación, responsabilidad, etc. y todo esto en un clima lúdico y de disfrute" (2008, p. 10).

Esta línea de pensamiento enfatiza la fuerte relación que se establece entre ciertos valores relacionados con el desarrollo personal, el descubrimiento y la educación social, los cuales crecen en espacios de práctica deportiva y perduran toda la vida (Ruiz y Cabrera, 2008).

Como toda acción humana, el deporte, la actividad física y el ejercicio físico se orientan y buscan el desarrollo de ciertos valores de carácter individual y social, que se traducen necesariamente en prácticas de comportamiento claramente identificables en los sujetos que los practican. Autores como Elizondo, Arazuri, Valdemoros y Echazarreta (2009), identifican la 
existencia de comportamientos que reflejan una valoración diferente de los practicantes hacia ciertas normas de carácter social y hacia los valores que las sustentan. Esta situación no solo afecta a aquellos que practican dichos deportes, sino también a los padres y profesores (agentes socializadores) que participan del proceso.

Según los mismos autores, se estima que la práctica físico-deportiva puede ser un espacio increíblemente favorecedor de la autoestima y el autoconcepto; no obstante, se intuye que, del mismo modo, podría constituirse en un medio capaz de minar la ilusión, la confianza y el deseo de continuar en esta experiencia cuando se concede mayor atención y reconocimiento a quienes destacan por sus resultados; de este modo, se fomentan sentimientos de aislamiento, marginación y desestima en quienes no se distinguen por sus aptitudes físico-deportivas.

En esta misma línea de pensamiento, Amat y Batalla plantean:

Hay muchos valores que pueden trabajarse mediante la práctica deportiva. Sin ánimo de ser exhaustivos, podemos hablar de valores utilitarios o ligados a la organización del trabajo (el esfuerzo, la dedicación, la entrega), de valores relacionados con la salud (el cuidado del cuerpo, la consolidación de hábitos alimentarios o higiénicos correctos) y de valores morales (la cooperación, el respeto de las normas) (2000, p. 2).

Uno de los aspectos que más destacan los citados autores es el cambio en la forma de entender los procesos de formación en valores:

En la actualidad no se concibe la educación en valores como la simple transmisión de valores socialmente aceptados, sino que lo que se pretende es propiciar que se den las condiciones que faciliten el que las personas construyan su propia jerarquía de valores, de tal manera que mantengan un equilibrio entre las necesidades propias y las colectivas, es decir, entre los ámbitos personal y comunitario-social (Amat y Batalla, 2000, p. 2).

Por otra parte, Gutiérrez, M. enfatiza: "para que el deporte favorezca la educación integral de la persona ha de practicarse en un clima apropiado en el que resulta especialmente importante la orientación de valores de técnicos, entrenadores, profesores y demás agentes socializadores" (2004, p. 122). 
Aspecto clave en esta reflexión es el de los procesos de socialización hacia el deporte (Boixadós et ál, 1998). La práctica de ejercicio físico inicia, para cada individuo, nuevas relaciones sociales que fomentan valores $\mathrm{y}$ actividades y generan nuevas experiencias y vivencias. Las diferentes investigaciones en este ámbito estudian cuáles son las influencias psicológicas y sociales que configuran la atracción inicial de los jóvenes por el deporte; estas influencias incluyen las actitudes y valores más relevantes dentro de la familia o del equipo. Diversas investigaciones de Cayuela Maldonado (1997) indican la existencia de tres elementos claves entre la realización de ejercicio físico y la formación en ciertos valores de carácter social: a) atributos personales de los individuos; b) agentes de socialización (pares, padres, entrenadores, docentes); c) situaciones y escenarios de socialización (familia, institución educativa, escenarios de entrenamiento y práctica deportiva, escenarios de competencia, etc.).

Por otra parte, la socialización a través del deporte refleja que, unidos a las condiciones de carácter personal que lleva a las personas a iniciarse en el deporte, los agentes socializadores y los escenarios de socialización son fundamentales. Los tres aspectos antes mencionados permiten el aprendizaje de actitudes y valores que no solo se ponen en ejercicio en escenarios y procesos deportivos, sino en otros muchos momentos de desenvolvimiento social.

En su revisión de antecedentes, Cayuela Maldonado (1997) indica que en las últimas décadas se han explorado posibles conexiones entre la participación deportiva, las actitudes y los comportamientos de los participantes en otros escenarios. Las hipótesis de los investigadores se basan en la creencia popular sobre las consecuencias de la participación deportiva en el desarrollo social. Según Coakley (1993) y Greendorfer (1992), citados por Cayuela Maldonado (1997), estos estudios reflejan la contribución de la práctica deportiva en la creación de un carácter positivo y demuestran cómo los deportistas pueden tener más posibilidad de éxito en la vida adulta en algunos escenarios específicos.

Sin embargo, estas investigaciones no han podido identificar en forma clara las consecuencias en los procesos de socialización de ciertas prácticas deportivas y, en este mismo orden de ideas, no identifican la relación concreta y directa entre tales procesos y la formación de valores sociales, ya que los individuos que practican deportes o realizan ejercicio físico no se encuentran aislados de otros escenarios de socialización donde se producen 
procesos de aprendizaje y formación social en valores. Estudios recientes priorizan en sus investigaciones otros aspectos más específicos, por ejemplo, cómo se origina la participación en el deporte y el desarrollo de habilidades sociales especialmente en los atletas de alto nivel.

\section{Conclusiones}

Los anteriores antecedentes evidencian que, durante la práctica de un deporte, se desarrollan hábitos y comportamientos asociados con el desarrollo de valores, no solo de tipo deportivo, sino directamente con valores y conductas valoradas como positivas a nivel social. Lo anterior indica que si se observan el deporte, el ejercicio físico y la actividad física en una línea del tiempo, su práctica continuada y constante impacta ciertos procesos formativos asociados al desarrollo de valores que (se esperaría) el individuo fortalezca con el tiempo y afecte otros escenarios de su vida, así no se convierta en un deportista profesional. Sin embargo, no son claros (por lo menos desde la revisión) los procesos de carácter educativo y formativo relacionados con los valores sociales que se dan en un escenario de acondicionamiento físico -el gimnasio-, y con la práctica del deporte y el ejercicio físico en un contexto universitario.

En el plano educativo, la reflexión sobre la relación entre el ejercicio físico y los valores se centra en identificar la importancia de la clase de educación física en el desarrollo y afianzamiento de valores, tanto en el plano individual como colectivo. De igual forma, los trabajos muestran una clara correspondencia entre los escenarios práctico y conceptual de las clases de educación física y la formación de valores.

A pesar de la diversidad de estudios y la aproximación de enfoques, psicólogos y sociólogos del deporte, todavía no se puede explicar cómo y por qué el aprendizaje social de ciertos valores sociales es mejor en unos practicantes que en otros. Lo que sí queda claro hasta ahora es que, junto con los agentes de socialización, aspectos como la estructura organizativa de las instituciones, la filosofía de los programas de formación, las orientaciones y conductas del entrenador (o del profesional que guía el ejercicio físico) afectan la experiencia deportiva y los valores de los jóvenes participantes. 
Es decir, el ambiente social bajo el que se realiza la práctica deportiva y el ejercicio son fundamentales.

Situaciones como el reconocimiento social positivo que tiene el ejercicio físico y su práctica, el fortalecimiento en la disciplina y el compromiso -tanto individual como colectivo-, la generación de contextos de valoración y reconocimiento social positivo -que necesariamente se producen dentro de los gimnasios- y la asociación que realizan los individuos entre el bienestar físico y mental que produce el ejercicio físico, además de sus obvias consecuencias en el plano biológico, se convierten en un elemento potenciador y de refuerzo positivo de la asociación entre la práctica del ejercicio y los valores que este desarrolla; la revisión de esta relación lo demuestra claramente. Sin embargo, la misma revisión presenta que, en el aspecto propiamente metodológico, tanto los instrumentos como las metodologías de acceso a los sujetos reducen la participación activa y los procesos de interpretación y reconstrucción de sentido que ellos hacen frente al reconocimiento social de dicha práctica y las implicaciones en la consolidación de ciertos valores sociales.

De igual forma, es claro que el énfasis de las investigaciones sobre esta relación se fortalece en métodos, técnicas e instrumentos de una clara tendencia positivista y bajo tipologías cuantitativas. Aunque es valiosa la capacidad de generalización que ofrecen, quedan cortas frente a procesos comprensivos e interpretativos de la realidad, enmarcada en un contexto histórico y social específico.

Por último, cabe resaltar que en la revisión específica para el caso colombiano, solo se encontró un trabajo de corte investigativo desarrollado en gimnasios universitarios, lo que fortalece la necesidad de aumentar el número, la calidad y el nivel de comprensión frente a diversos procesos sociales y culturales asociados a la práctica del ejercicio físico en dichos escenarios.

\section{Referencias}

Álvarez, A. (2003). Estudio del pensamiento de los entrenadores sobre el proceso de detección de talentos en baloncesto. European Journal of Human Movement, $10,23-51$. 
Amat, M. y Batalla, A. (2000). Deporte y educación en valores. Revista Candidus 1, (12). Recuperado de: www.revistacandidus.com

Boixadós, M. Valiente, L., Mimbrero, J., Torregrosa, M. y Cruz, J. (1998). Papel de los agentes de socialización en deportistas en edad escolar. Revista de Psicología del Deporte, 7(2), 295-310.

Cayuela Maldonado, M. J. (1997). Los efectos sociales del deporte: ocio, integración, socialización, violencia y educación. Barcelona: Centre d'Estudis Olímpics UAB. Recuperado de: http://olympicstudies.uab.es/pdf/wp060_spa.pdf

Delgado, R. (2007). Los marcos de acción colectiva y sus implicaciones culturales en la construcción de ciudadanía. Revista Universitas Humanística, 64, 41-66. Recuperado de: http://www.fundacionasciende.com/publicaciones -de-fundacion-asciende/articulos/28-valores-morales-en-el-deporte

Ferrater Mora, J. (1994). Diccionario de Filosofía. Barcelona: Ariel.

Flores M., R. y Zamora, J. D. (2009). La educación física y el deporte como medios para adquirir y desarrollar valores en el nivel de primaria. Revista Educación, 33(1), 133-143.

Gallardo, I. (2008). Valores morales en el deporte: hacia la detección del papel del deporte como agente socializador y catalizador de la internalización de las normas y valores morales. Deporte, Salud, Educación y Desarrollo, 1-10. Fundación Asciende. Recuperado de http://www.fundacionasciende.com/publi caciones-de-fundacion-asciende/articulos/28-valores-morales-en-el-deporte

Gómez Rijo, A. (2005). La enseñanza y el aprendizaje de los valores en la educación deportiva. Revista Internacional de Medicina y Ciencias de la Actividad Física y el Deporte, 5(18), 89-99.

Gutiérrez, M. (2004). El valor del deporte en la educación integral del ser humano. Revista de Educación, 335, 106. España: Universidad de Valencia.

Gutiérrez, M. L. (2004). Una mirada al ejercicio físico en los gimnasios de Medellín desde la promoción de la salud y prevención de la enfermedad primaria. Tesis de especialización. Medellín: Universidad de Antioquia, Instituto Universitario de Educación Física.

Gutiérrez, S. M. (2003). Manual sobre valores en la educación física y el deporte. Buenos Aires: Paidós.

Hoyos, G. y Vargas, G. (1996). La teoría de la acción comunicativa como nuevo paradigma de investigación en ciencias sociales: las ciencias de la discusión. Módulo 2. Bogotá: Icfes. 
Morse, J. (2003). Asuntos críticos en los métodos de investigación cualitativa. Medellín: Universidad de Antioquia.

Olmedilla, A., Ortega, E., Garcés de los Fayos, E., Jara, P. y Ortín, F. (2009). Evolución de la investigación y de la aplicación en psicología del deporte, a través del análisis de los congresos nacionales de psicología del deporte (19992008). Cultura, Ciencia y Deporte, 5(4), 15-23. Universidad Católica San Antonio de Murcia.

Pardo, R. (2008). La transmisión de valores a jóvenes socialmente desfavorecidos a través de la actividad física y el deporte. Tesis doctoral europea. Universidad Politécnica de Madrid, Red de Información Educativa. Id: 38663324.

Ponce de León Elizondo, A., Sanz Arazuri, E., Valdemoros San Emeterio, M. A. y Ramos Echazarreta, R. (2009). Los valores personales en el ocio físico-deportivo: un estudio con jóvenes, padres y profesores. Revista de Pedagogía, 61(1), 29-42. Bordón.

Ruiz, G. y Cabrera, D. (2008). Los valores en el deporte. Revista de Educación, 335, 9-19. Universidad de Málaga.

Souza, M. S. (2008). La centralidad del estado del arte en la construcción del objeto de estudio. Facultad de Periodismo y Comunicación Social de la Universidad Nacional de La Plata, apunte de Cátedra. Recuperado de: http://perio.unlp.edu. ar/seminario/nivel2/nivel3/textos_actualizados_2008/La\%20centralidad\%20 de1\%20estado\%20de1\%20arte\%20en\%201a\%20construccin\%20de1\%20objeto $\% 20$ de $\% 20$ estudio.pdf

Taylor, S.J. y Bogdan, R. (1987). Introducción a los métodos cualitativos de investigación. España: Paidós Básica.

Torregrosa, M. y Lee, M. (2009). El estudio de los valores en psicología del deporte. Revista de Psicología del Deporte, 9(1-2), 71-83. 